\title{
Commentary
}

\section{miR-634 inhibits human vascular smooth muscle cell proliferation and migration in hypertension through Wnt4/ $\beta$-catenin pathway}

\author{
Ligang $\mathrm{Niu}^{1}$, Na Sun${ }^{2}$, Lingheng Kong ${ }^{2}$, Yan $\mathrm{Xu}^{2}$, Yuming Kang ${ }^{3, *}$ \\ ${ }^{1}$ Department of Breast Surgery, The First Affiliated Hospital of Xi'an Jiaotong University, 710061 Xi'an, Shaanxi, China, \\ ${ }^{2}$ Department of Physiology, Xi'an Medicine University, 710021 Xi'an, Shaanxi, China, ${ }^{3}$ Department of Physiology and \\ Pathophysiology, Xi'an Jiaotong University School of Basic Medical Sciences, Shaanxi Engineering and Research Center \\ of Vaccine, Key Laboratory of Environment and Genes Related to Diseases of Education Ministry of China, 710061 Xi'an, \\ Shaanxi, China
}

\section{TABLE OF CONTENTS}

\section{Abstract}

2. Introduction

3. Materials and methods

\author{
3.1 Study population \\ 3.2 Cell culture and treatment \\ 3.3 Cell transfection \\ 3.4 Proliferation assay \\ 3.5 Transwell assay \\ 3.6 Luciferase reporter assay \\ 3.7 qRT-PCR \\ 3.8 Western blot \\ 3.9 Statistical analysis
}

4. Results

4.1 miR-634 was reduced in Ang II-induced HASMCs

4.2 miR-634 suppressed Ang II-induced HASMCs proliferation and migration

4.3 Wnt4 was a target gene of miR-634

4.4 Over-expression of Wnt4 counteracted the suppressive effects of miR-634 on Ang II-induced HASMCs

proliferation and migration

4.5 miR-634 was reduced in hypertensive patients

5. Discussion

6. Conclusions

7. Author contributions

8. Ethics approval and consent to participate

9. Acknowledgment

10. Funding

11. Conflict of interest

12. Availability of data and materials

13. References

\section{Abstract}

MicroRNAs (miRNAs) have been regarded as modulators in vascular pathologies, including hypertension. Dysregulated proliferation and migration of VSMCs (vascular smooth muscle cells) contributes to vascular remodeling during hypertension. miR-634 was reported to be dysregulated in hypertensive patients. The involvement of miR-634 in hypertension and the role of miR-634 on VSMCs proliferation and migration were then evaluated. Firstly, HASMCs (human aortic smooth muscle cells) were incubated with $2 \mu \mathrm{M}$ angiotensin (Ang) II for 12 hours to establish the cell model of Ang II-induced hypertension. 
Results showed that Ang II treatment promoted proliferation and migration of HASMCs. Secondly, miR-634 was down-regulated in the hypertensive patients, and reduced in Ang II-induced HASMCs in a time dependent manner. Functional assays revealed that Ang II promoted proliferation and migration of HASMCs were suppressed by miR634 mimic. Lastly, miR-634 targeted 3' untranslated region (UTR) of Wnt4, and reduced Wnt4 expression in HASMCs. miR-634 inhibited $\beta$-catenin nuclear translocation. Overexpression of Wnt4 counteracted the suppressive effects of miR-634 on Ang II-induced proliferation and migration of HASMCs. In conclusion, miR-634 inhibited HASMCs proliferation and migration through inactivation of Wnt $4 / \beta$ catenin pathway.

\section{Introduction}

Hypertension causes cardiovascular diseases and is considered as a world-wide disease [1]. The etiology of hypertension is complicated with various pathogenic factors. Environmental and genetic factors could lead to increased blood pressure through affecting physiological processes [2], thus leading to hypertension [3]. Overreaction of renin-angiotensin-aldosterone system, dysfunction of vascular endothelial cells, cardiac hypertrophy and platelet function injury have been widely regarded as pathogenesis of hypertension [4]. Meanwhile, vascular remodeling, associated with dysregulated proliferation and migration of VSMCs, is the central mechanism for hypertension [5]. Therefore, inhibition of VSMCs proliferation and migration represents an effective therapeutic strategy for hypertension [6].

miRNA, or microRNAs, are small non-coding RNAs with about 19-23 nucleotides in length in a class of organisms [7]. It has been reported that miRNAs could be involved in multiple pathological processes, including VSMCs proliferation and migration, through targeting $3^{\prime}$ untranslated region (UTR) of mRNAs [7]. Moreover, miRNAs are closely related to vascular remodeling and endothelial cell growth regulation [8]. Increasing evidence has shown that miRNAs could control endothelial response to regulate the secretion of inflammatory and pro-coagulant mediators, thus regulating blood pressure during hypertension [9]. Therefore, miRNAs are reported to be implicated in the pathogenesis of hypertension and may play an important regulatory role in the occurrence and development of hypertension. For example, high-salt diet could lead to the up-regulation of miR-29b in the renal medulla of rats and miR-29b can regulate the expression of collagen genes and related genes in vascular endothelial cells, thus regulating salt-sensitive hypertension [10]. Decreased expression of miR-143 and miR-145 could lead to the decrease in the number of vascular smooth muscle cells and vasoconstriction force, resulting in the decrease in blood pressure and amelioration of vascular disease [11]. Recently, studies have found that miR-634 was down-regulated in idiopathic pulmonary hypertension patients [12]. In addition, miR634 was also reduced in hypertensive patients [13]. However, the regulatory role of miR-634 in the pathogenesis of hypertension remains elusive.

$\mathrm{Wnt} / \beta$-catenin, a developmental signal pathway, is involved in injury repair and tissue homeostasis [14]. In heart tissue, Wnt signal is relatively silent, while activated after cardiac injuries [15]. Dysregulation of Wnt $/ \beta$-catenin was involved in cardiac hypertrophy [16]. $\beta$-catenin is also involved in renal fibrosis of hypertensive rats [17]. Moreover, hyperactive $\mathrm{Wnt} / \beta$-catenin could promote the activation of renin-angiotensin system and contribute to hypertension [18]. Wnt4 was considered as a noninvasive biomarker of renal injury post hypertension [19]. Therefore, Wnt4 may be a potential target for the treatment of hypertension. In addition, miR-634 was down-regulated in Dupuytren's contracture and participated in deregulation of $\beta$-catenin pathway [20]. This study was then conducted to validate the speculation that miR-634 regulated VSMCs proliferation and migration during hypertension through $\mathrm{Wnt} / \beta$-catenin pathway.

\section{Materials and methods}

\subsection{Study population}

All procedures involved in human subjects were approved by the Ethics Committee of the First Affiliated Hospital of Xi'an Jiaotong University according to those of the 1964 Helsinki Declaration and its later amendments for ethical research involving human subjects. Forty-one health people with systolic blood pressure $<120 \mathrm{mmHg}$ and diastolic blood pressure $<80 \mathrm{mmHg}$ were recruited at the First Affiliated Hospital of Xi'an Jiaotong University. Sixty-eight hypertensive patients with systolic blood pressure $\geq 140 \mathrm{mmHg}$ and/or diastolic blood pressure $\geq 90$ mmHg were recruited at the First Affiliated Hospital of Xi'an Jiaotong University. Written informed consents were obtained from all the human subjects. Patients who had cognitive impairment or incomplete tests or data were excluded in this study. Three mL peripheral blood from all the human subjects was collected in tubes containing EDTA. The samples were then centrifuged to separate the plasma at $1000 \mathrm{~g}$ for 20 minutes. RNAs were extracted from the plasma via mirVana ${ }^{\mathrm{TM}}$ PARIS ${ }^{\mathrm{TM}}$ kit (Ambion, Austin, TX, USA) for the subsequently analysis. The clinical characteristics of patients with hypertension were shown in Table 1.

\subsection{Cell culture and treatment}

HASMCs (human aortic smooth muscle cells) or human vascular smooth muscle cells (HVSMCs) were acquired from Lonza (Rockland, ME, USA) and cultured in Smooth Muscle Cell Medium (SciencCell, Carlsbad, CA, USA) supplemented with $10 \%$ fetal bovine serum (Lonza) at $37{ }^{\circ} \mathrm{C}$ humidified incubator with $5 \% \mathrm{CO}_{2}$. For the es- 
Table 1. Clinical characteristics of patients with hypertension.

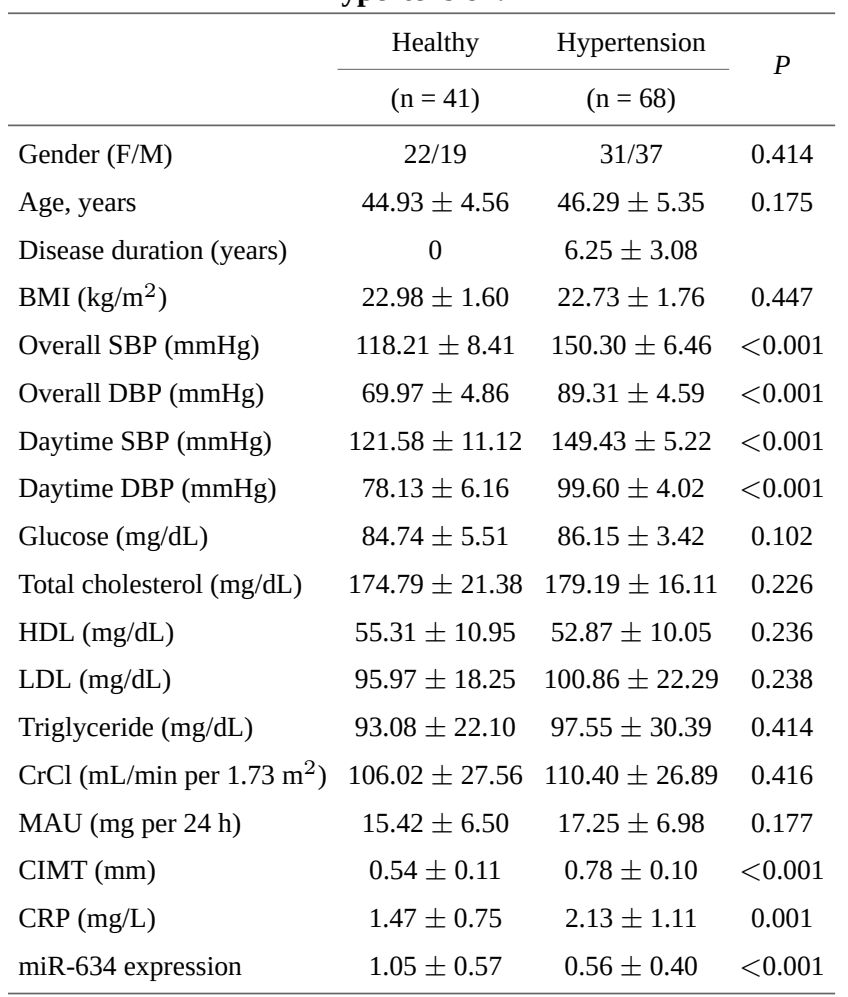

Pearson chi-square test is for Gender and $T$ test for others.

BMI, body mass index; SBP, systolic blood pressure; DBP, diastolic blood pressure; HDL, high density lipoprotein; LDL, low density lipoprotein; $\mathrm{CrCl}$, creatinine clearance; MAU, microalbuminuria; CIMT, carotid intima media thickness; CRP, C-reactive protein.

tablishment of Ang II-induced hypertension cell model, HASMCs or HVSMCs were cultured in Smooth Muscle Cell Medium containing $2 \mu \mathrm{M}$ Ang II for 12 hours before the functional assays. For the treatment of Ang II, HASMCs were cultured in Smooth Muscle Cell Medium containing $2 \mu \mathrm{M}$ Ang II and 200 or $500 \mathrm{ng} / \mathrm{mL}$ human Wnt4 recombinant protein (R\&D Systems, Minneapolis, MN, USA) for 12 hours before the functional assays.

\subsection{Cell transfection}

Mimic (5'-AACCAGCACCCCAACUUUGGAC GGTATTCGCACTGGATACGACGAACTTT-3') and inhibitor (5'-CACUACUUUUGUGUCCCACUU-3') of miR-634, as well as the corresponding negative controls (NC mimic, NC inhibitor), were acquired from Ribobio (Guangzhou, China). Full length of Wnt4 was constructed into pcDNA3.1 vector (Invitrogen, Carlsbad, CA, USA). HASMCs were transfected with miR-634 mimic, inhibitor, NC mimic, NC inhibitor, pcDNA-Wnt4 or pcDNA vector by Lipofectamine 3000 (Invitrogen). Twenty-four hours later, cells were treated with or without $2 \mu \mathrm{M}$ Ang II, 200 or $500 \mathrm{ng} / \mathrm{mL}$ human Wnt4 recombinant protein for 12 hours before the functional assays.

\subsection{Proliferation assay}

HASMCs (4000 cells/well) were seeded and then treated with or without different treatment or transfection. After the different treatment, cultured medium of HASMCs were changed to fresh medium containing $50 \mu \mathrm{M}$ EdU (Sigma-Aldrich, St. Lou-is, MO, USA) for 4 hours. Cells were then fixed in 4\% paraformaldehyde, and incubated with EdU antibody for 2 hours. After washing with phosphate-buffered saline, cells were incubated with 200 $\mu \mathrm{L}$ Apollo staining reaction buffer. The immunostaining was in 5 randomly selected fields measured under fluorescence microscopy (Nikon, Tokyo, Japan) with nuclei staining with DAPI.

\subsection{Transwell assay}

HASMCs (30000 cells/well) in serum-free medium were seeded to the top of the chamber with 8 $\mu \mathrm{m}$ pore size (Coatar, Cambridge, MA, USA). Cultured medium containing $20 \%$ fetal bovine serum was added to the low chamber. Twenty-four hours after incubation, migrating cells to the low chamber were washed, fixed and stained with crystal violet. Cells in 5 randomly selected fields were measured under microscope (Nikon) using Image J (NIH, Bethesda, MD, USA).

\subsection{Luciferase reporter assay}

The 3' UTR of Wnt4 containing miR-634 binding site or mutant control without the binding site were subcloned into pGL3 vector (GenePharma, Suzhou, China). HASMCs (20000 cells/well) were seeded and then cotransfected with pRL-TK (10 ng), miR-634 mimic or NC mimic (20 nM) and the pGL3 vectors (100 ng). Luciferase activities were performed via Dual luciferase reporter assay kit (Promega, Madison, WI, USA) 48 hours after transfection.

\section{7 qRT-PCR}

Plasma RNAs, miRNAs or RNAs extracted from HASMCs were isolated and reverse-transcribed into cDNAs. qRT-PCR analysis was conducted with QuantiTect SYBR Green PCR Master Mix (Qiagen, Valencia, CA, USA). GAPDH or U6 were used as endogenous controls. The primer sequences were showed as below: miR-634 (F: 5'-ATTATGTTAGTTAGGATGGTTTCGA3'; R: 5'-ATATCCACAAACAAATAACTTCGTT-3'); Wnt4 (F: 5' $5^{\prime}$-AGGAAGCCAGGTAAAGTGCTC3'; R: 5'-CAGCCAGACCACTCAAAGTTG-3'); GAPDH (F: 5'-ACCACAGTCCATGCCATCAC3'; R: 5'-TCCACCACCCTGTTGCTGTA-3'); U6 (F: 5'-CTCGCTTCGGCAGCACATA-3'; R: 5'AACGATTCACGAATTTGCGT-3'). 
A
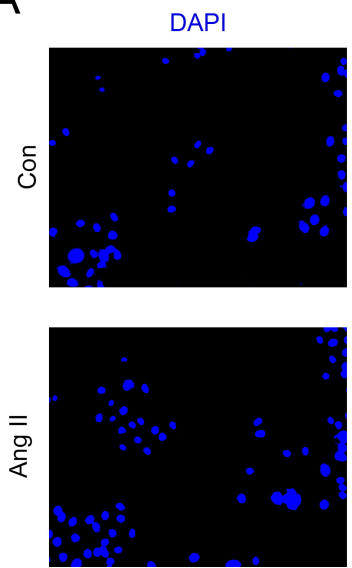

Edu
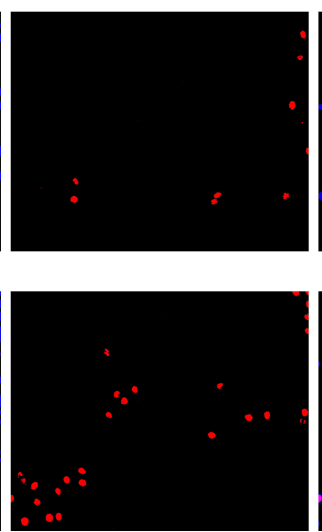

$12 \mathrm{~h}$
B
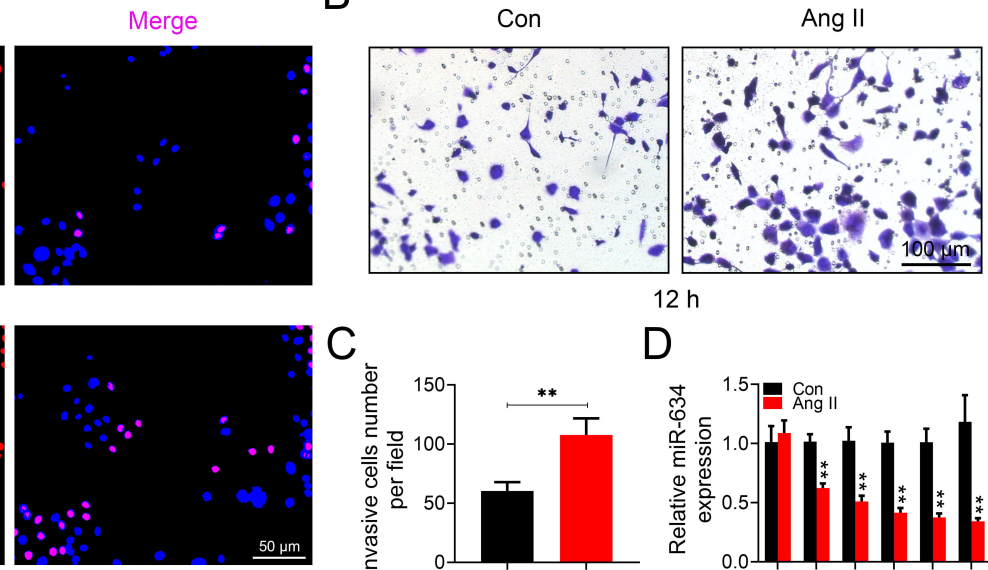

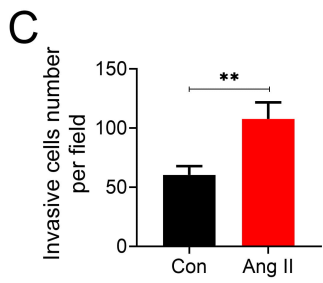

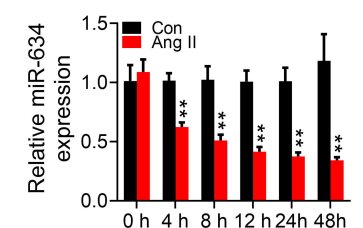

Fig. 1. miR-634 was reduced in Ang II-induced HASMCs.

(A) The effect of $2 \mu \mathrm{M}$ Ang II on cell proliferation of HASMCs was detected by EdU staining. (B) The effect of $2 \mu \mathrm{M}$ Ang II on cell migration of HASMCs was detected by transwell. (C) Invasion cells number affected by Ang II treatment. (D) The effect of $2 \mu \mathrm{M}$ Ang II on miR-634 expression of HASMCs in different time. ${ }^{* *} p<0.01$. Scale bars: $50 \mu \mathrm{m}$.

\subsection{Western blot}

For isolation of cytoplasm and nuclear fractionation, HASMCs were harvested and suspended in isolation buffer A containing protease inhibitors (HiScript II First Strand cDNA Synthesis Kit; Vazyme Biotech, Nanjing, Jiangsu, China). After rotating for 1 minute and centrifuging at $12000 \mathrm{~g}$ for 5 minutes, supernatant with cytoplasm fraction was collected. The debris was suspended in isolation buffer B containing protease inhibitors, and then rotated for the collection of nuclear fractionation. For western blot analysis, proteins (30 $\mu \mathrm{g}$ ) extracted from HASMCs were subjected to sodium dodecyl sulfate-polyacrylamide gel electrophoresis, and then electro-transferred onto nitrocellulose membrane. Membranes were blocked in 5\% skim milk, and incubated with primary antibodies: antiWnt4 (1:1500; Abcam, Cambridge, MA, USA), anti- $\beta$ catenin (1:2500; Abcam, Cambridge, MA, USA), antiHistone, anti-GAPDH and anti- $\beta$-actin (1:3000; Abcam, Cambridge, MA, USA). After incubation with horseradish peroxidase labeled secondary antibody (1:5000; Abcam, Cambridge, MA, USA), the signals were determined by Amersham enhanced chemiluminescence detection system (GE Healthcare Life Sciences, Little Chalfont, UK).

\subsection{Statistical analysis}

All the experiments were performed at least in triplicates. Data were expressed as mean $\pm S D$, and performed by SPSS 19.0 software (SPSS, Chicago, IL, USA). Statistical analysis was determined by Student's $t$-test or one-way analysis of variance. $p$ value $<0.05$ was considered to be statistically significant.

\section{Results}

\section{1 miR-634 was reduced in Ang II-induced HASMCs}

To establish cell model of hypertension, HASMCs were incubated with culture medium containing $2 \mu \mathrm{M}$ Ang II for 12 hours. Ang II treatment promoted HASMCs proliferation, as demonstrated by increased EdU incorporation (Fig. 1A). Ang II treatment also promoted migration of HASMCs by (Fig. 1B), as shown by increased cell migration in HASMCs treated with Ang II compared with the control (Fig. 1C), suggesting successful cell model of hypertension. miR-634 was down-regulated in Ang IIinduced HASMCs in a time dependent manner (Fig. 1D), suggesting that miR-634 might be involved in regulation of HASMCs proliferation and migration.

\section{2 miR-634 suppressed Ang II-induced HASMCs proliferation and migration}

To evaluate the role of miR-634 in HASMCs proliferation and migration, HASMCs or HVSMCs were transfected with miR-634 mimic and then treated with Ang II. qRT-PCR confirmed the up-regulation of miR-634 in HASMCs (Fig. 2A) and HVSMCs (Supplementary Fig. 1A) transfected with miR-634 mimic. Over-expression of miR-634 suppressed cell proliferation (Fig. 2B) and migration (Fig. 2C,D) of HASMCs, as evidenced by the decreased EdU incorporation (Fig. 2B) and invasion cells number (Fig. 2D) in HASMCs transfected with miR634 mimic compared with cells transfected with negative control. Moreover, transfection with miR-634 mimic suppressed the cell proliferation (Fig. 2B) and migration (Fig. 2C,D) of Ang II-induced HASMCs, suggesting that miR-634 might contribute to amelioration of hypertension. 


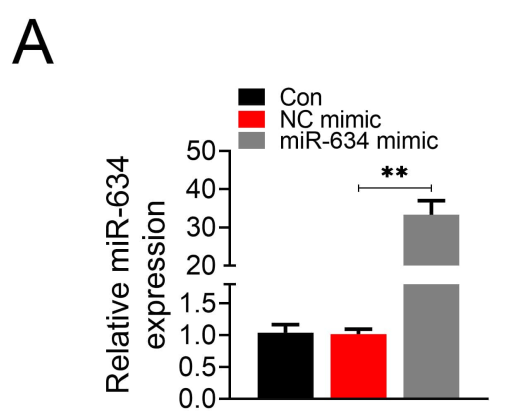

B

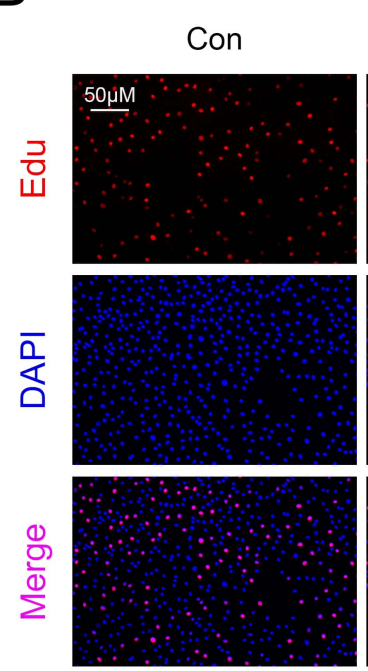

C

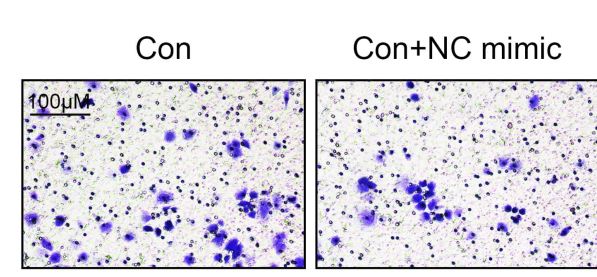

Con+NC mimic
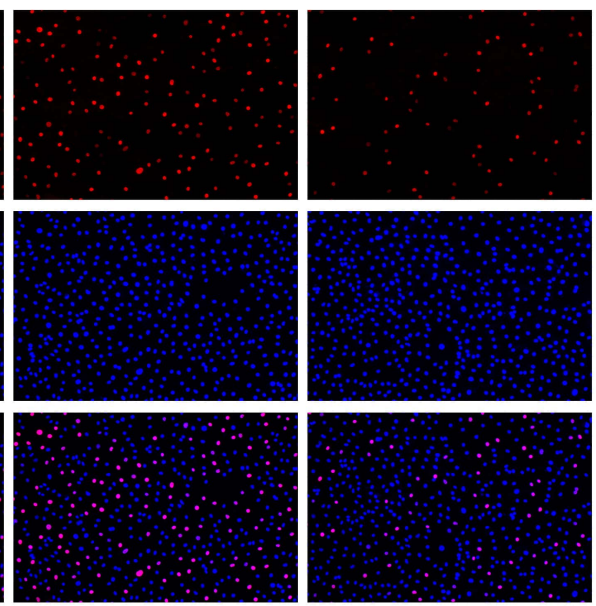

Con+ miR-634 mimic

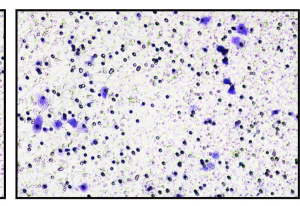
miR-634 mimic

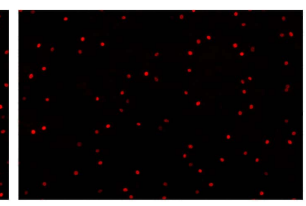

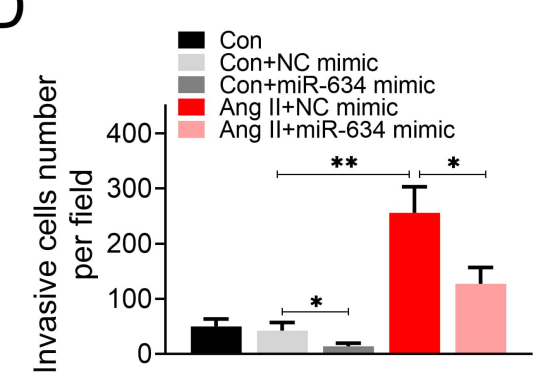

Con+

Ang II+NC mimic

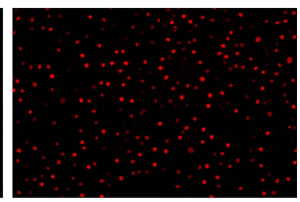

miR-634 mimic
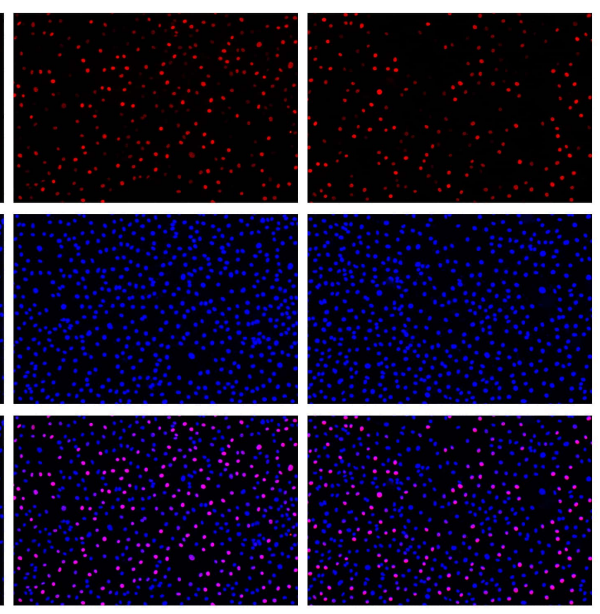

Ang II+ Ang II+NC mimic miR-634 mimic

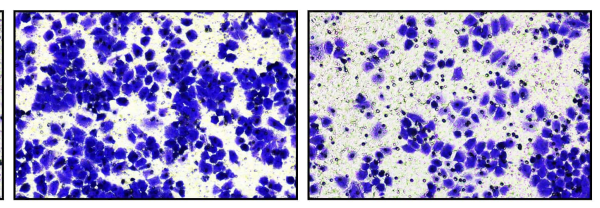

Fig. 2. miR-634 suppressed Ang II-induced HASMCs proliferation and migration.

(A) Transfection efficiency of miR-634 mimic in HASMCs was detected by qRT-PCR. (B) Effect of miR-634 on cell proliferation of HASMCs with or without Ang II treatment. (C) Effect of miR-634 on cell migration of HASMCs with or without Ang II treatment. (D) Effect of miR-634 on invasion cells number of HASMCs with or without Ang II treatment. *, **, $p<0.05, p<0.01$. NC mimic: negative control of miR-634 mimic. Scale bars: $50 \mu$ m.

The cell proliferation (Supplementary Fig. 1B) and migration (Supplementary Fig. 1C,D) of HVSMCs were also promoted by Ang II treatment, while reduced by overexpression of miR-634.

\subsection{Wnt4 was a target gene of miR-634}

To investigate the mechanism of miR-634 in HASMCs, the target gene of miR-634 was predicted as Wnt4 via Targetscan (http://www.targetscan.org/vert_72/) (Fig. 3A). Luciferase activity of pGL3-WT-Wnt4 was decreased in HASMCs transfected with miR-634 mimic compared with the negative control (Fig. 3B), while activity of pGL3-MUT-Wnt4 was not affected by miR-634 mimic compared with the negative control (Fig. 3B), suggesting that miR-634 could target Wnt4 in HASMCs. To vali- date effect of miR-634 on Wnt4 expression, HASMCs were transfected with miR-634 mimic or inhibitor (Fig. 3C). Results revealed that mRNA (Fig. 3D) and protein (Fig. 3E) of Wnt4 were reduced by miR-634 mimic, while enhanced by the inhibitor. Moreover, Wnt4 was upregulated in HASMCs after 12 hours of Ang II treatment (Supplementary Fig. 2A,B). These results indicated that miR-634 could target Wnt4 and repress its expression.

\subsection{Over-expression of Wnt4 counteracted the suppressive effects of miR-634 on Ang II-induced HASMCs proliferation and migration}

To investigate the role of miR-634/Wnt4 axis in HASMCs, Ang II-induced HASMCs were transfected with pcDNA-Wnt4 or cotransfected with miR-634 mimic and 
A

Position 2253-2260 of WNT4

3' UTR WT

hsa-miR-634

3' UTR MUT
5' ...UAGGUCCAGGCUGGAUGCUGGUA... 3'

3' CAGGUUUCAACCCCACGACCAA 5'
B

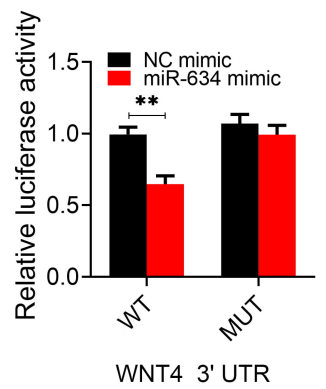

C

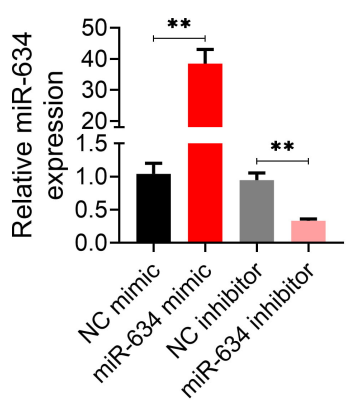

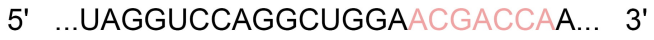
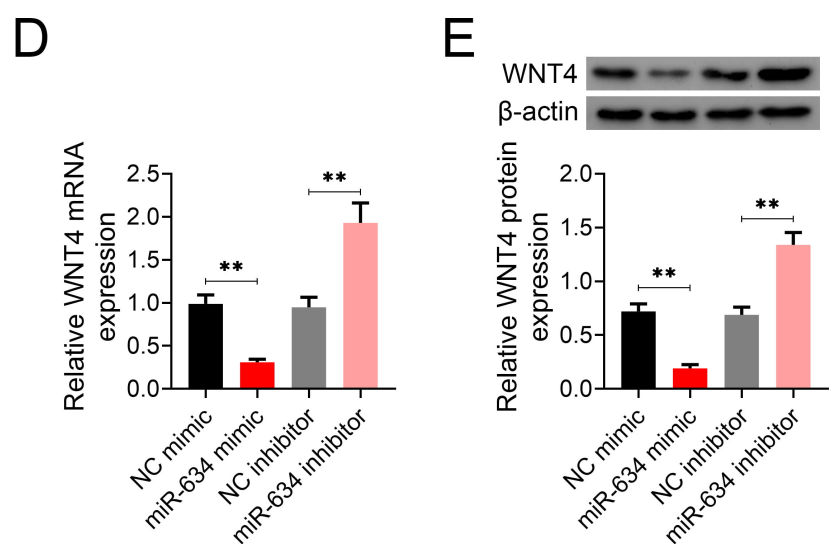

Fig. 3. Target gene of miR-634

(A) Potential binding target of miR-634 was predicted as Wnt4. (B) The effect of miR-634 on luciferase activities of pGL3-WT-Wnt4 and pGL3-MUTWnt4. (C) Transfection efficiency of miR-634 mimic or inhibitor in HASMCs was detected by qRT-PCR. (D) Effect of miR-634 on mRNA expression of Wnt4 in HASMCs. (E) Effect of miR-634 on protein expression of Wnt4 in HASMCs. ** $p<0.01$. NC inhibitor: negative control of miR-634 inhibitor.

pcDNA-Wnt4. The transfection efficiency of pcDNAWnt4 was shown in Supplementary Fig. 3. Results showed that over-expression of Wnt4 aggravated the promotive effects of Ang II on cell proliferation (Fig. 4A) and migration (Fig. 4B,C). However, cotransfection with miR-634 mimic and pcDNA-Wnt4 attenuated the promotive effects of Wnt4 on Ang II-induced HASMCs proliferation (Fig. 4A) and migration (Fig. 4B,C). These results showed that miR-634/Wnt4 axis participated in regulation of Ang II-induced HASMCs proliferation and migration. As a downstream target of Wnt4, nuclear distribution of $\beta$-catenin (Fig. 4D) was decreased in Ang II-induced HASMCs transfected with miR-634 mimic, while the cytoplasmic distribution was increased (Fig. 4D). Moreover, over-expression of Wnt4 enhanced the nuclear distribution of $\beta$-catenin (Fig. 4D) while decreased the cytoplasmic distribution (Fig. 4D). Furthermore, over-expression of Wnt4 reversed the inhibitory effect of miR-634 on nuclear distribution of $\beta$-catenin (Fig. 4D) and the promotive effect on the cytoplasmic distribution (Fig. 4D). The subcellular distribution of $\beta$-catenin indicated that function of miR$634 / \mathrm{Wnt}$ axis was dependent on $\beta$-catenin pathway. In vitro treatment with Wnt4 protein significantly promoted cell proliferation (Fig. 4E) and migration (Fig. 4F) in a dosedependent manner, as demonstrated that $500 \mathrm{ng} / \mathrm{mL}$ Wnt4 treatment showed more EdU incorporation (Fig. 4E) and migration cells (Fig. 4G) of Ang II-induced HASMCs than
$200 \mathrm{ng} / \mathrm{mL}$ Wnt4 treatment. Moreover, nuclear distribution of $\beta$-catenin (Fig. $4 \mathrm{H}$ ) was promoted by Wnt 4 treatment, while cytoplasmic distribution (Fig. 4G) was suppressed. Therefore, miR-634 could decrease Wnt 4 to reduce nuclear distribution of $\beta$-catenin, thus suppressing Ang II-induced HASMCs proliferation and migration.

\section{5 miR-634 was reduced in hypertensive patients}

Expression level of miR-634 in hypertensive patients was evaluated by qRT-PCR. Plasma samples from healthy individuals or hypertensive patients were analyzed and the result showed a significant decrease in miR-634 in hypertensive patients compared with health individuals $(p$ $<0.01$, Fig. 5). Analysis between miR-634 expression and clinical characteristics of patients with hypertension was shown in Table 1, suggesting that miR-634 might regulate hypertension.

\section{Discussion}

miRNA regulate renal function to maintain normal blood pressure [21]. Extracellular vesicles secreted by miR27a promoted blood pressure and led to hypertension [22]. miRNA target VSMCs to mediate vascular resistance or remodeling for the development of hypertension [23]. For example, reduction of miR-34a resulted in increased proliferation of human pulmonary artery smooth muscle cells 
A

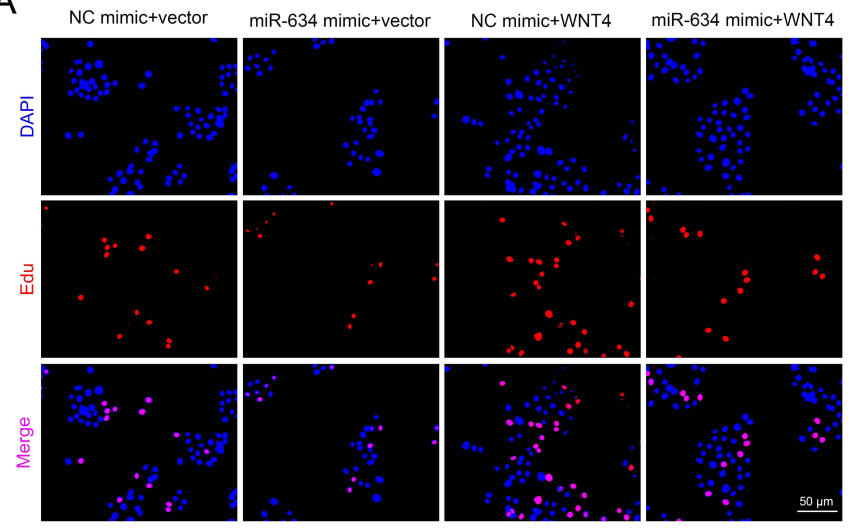

B

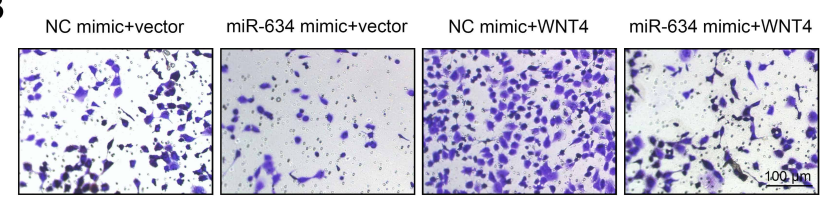

C

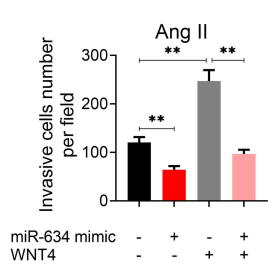

D

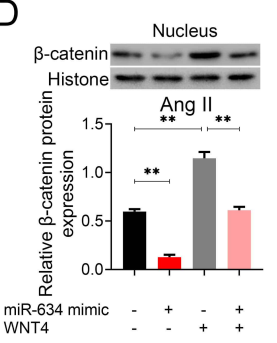

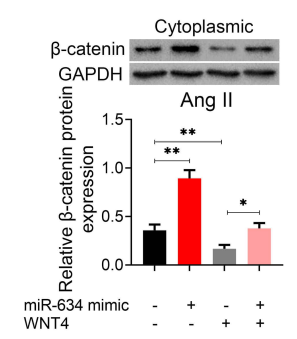

E

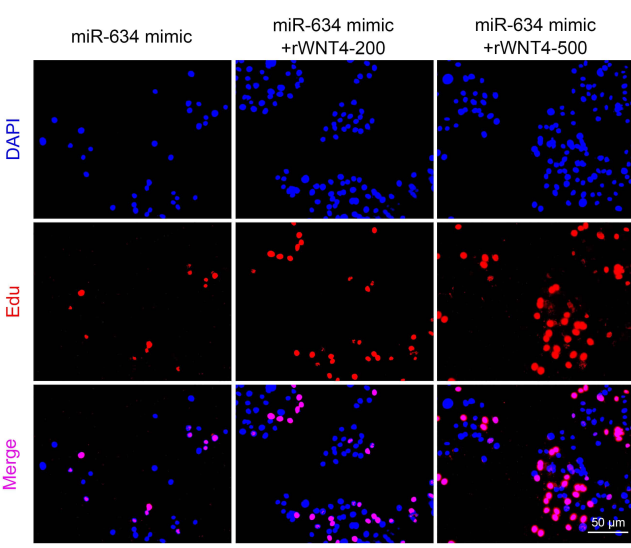

F

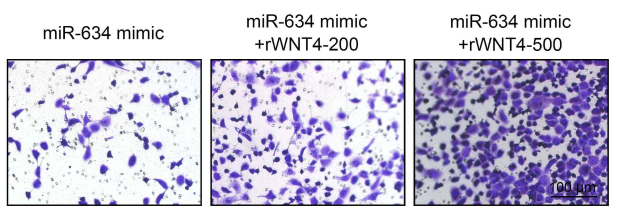

G

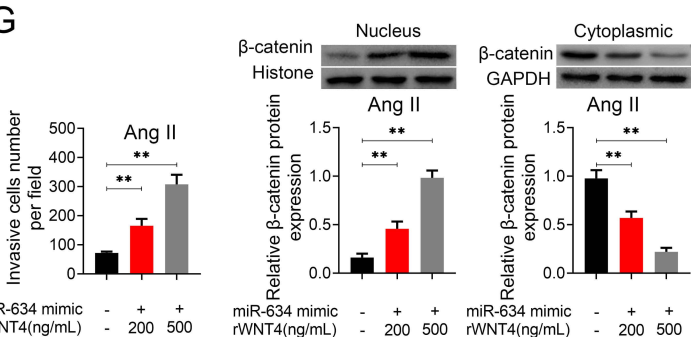

Fig. 4. Over-expression of Wnt4 counteracted the suppressive effects of miR-634 on Ang II-induced HASMCs proliferation and migration.

(A) Effect of miR-634 mimic and pcDNA-Wnt4 on cell proliferation of HASMCs with Ang II treatment. (B) Effect of miR-634 mimic and pcDNA-Wnt4 on cell migration of HASMCs with Ang II treatment. (C) Effect of miR-634 mimic and pcDNA-Wnt4 on cell migration of HASMCs with Ang II treatment. (D) Effect of miR-634 mimic and pcDNA-Wnt4 on nucleus and cytoplasmic distribution of $\beta$-catenin in HASMCs with Ang II treatment. (E) The effect of in vitro treatment with 200 or $500 \mathrm{ng} / \mathrm{mL}$ Wnt4 protein on cell proliferation of miR-634 mimic-transfected HASMCs under Ang II treatment. (F) The effect of in vitro treatment with 200 or $500 \mathrm{ng} / \mathrm{mL}$ Wnt4 protein on cell migration of miR-634 mimic-transfected HASMCs under Ang II treatment. (G) The effect of in vitro treatment with 200 or $500 \mathrm{ng} / \mathrm{mL}$ Wnt4 protein on invasion cells number of miR-634 mimic-transfected HASMCs under Ang II treatment. (H) The effect of in vitro treatment with 200 or $500 \mathrm{ng} / \mathrm{mL}$ Wnt4 protein on nucleus distribution and decreased cytoplasmic distribution of $\beta$-catenin in miR-634 mimic-transfected HASMCs under Ang II treatment. ${ }^{* *} p<0.01$. Vector: pcDNA-3.1 vector. Scale bars: $50 \mu \mathrm{m}$.

and contributed to pulmonary arterial hypertension development [24]. Over-expression of miR-17-5p regulated cell proliferation of human pulmonary artery smooth muscle cell through targeting arginase II [25]. Abrogation of pulmonary artery smooth muscle cells proliferation and migration by miR-233 led to the attenuation of vascular remodeling and pulmonary arterial hypertension [26]. However, whether miR-634 is associated with VSMCs proliferation and migration, thus regulating hypertension, remains unclear.

Previous study has indicated that miRNAs could be dysregulated in plasma samples or renal tissues of hypertensive patients, thus representing biomarkers of hypertension [27]. Down-regulation of miR-634 in idiopathic pulmonary hypertension patients [12] or hypertensive patients [13] have also been reported. Data from the present study revealed that miR-634 was down-regulated in the plasma of hypertensive patients. However, the smaller sample size, as well as devoid of clinicopathological correlation between miR-634 expression and hypertensive patients, restricted the use of circulating miR-634 as biomarker for hypertensive patients.

Ang II, a peptide hormone with vasoconstrictor effect, was elevated in plasma of essential hypertension [28]. Ang II could induce phenotypic transformation of VSMCs [29], and promote proliferation and migration of VSMCs, leading to vascular remodeling for the development of hypertension [30]. Therefore, Ang II-induced HASMCs has been widely used as a cell model of hypertension [31]. Our results also showed increased HASMCs proliferation and migration by Ang II treatment. Moreover, miR-634 was reduced in Ang II-induced HASMCs 


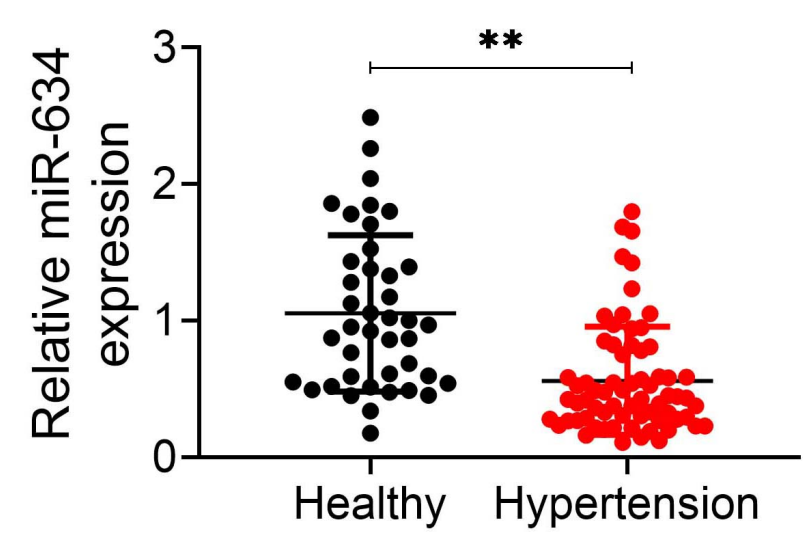

Fig. 5. miR-634 was reduced in hypertensive patients.

Expression level of miR-634 in hypertensive patients and healthy individuals. ${ }^{* *} p<0.01$.

in a time-dependent manner, suggesting the potential role of miR-634 in VSMCs.

Inhibition of Ang II-induced VSMCs proliferation and migration could facilitate the amelioration of hypertension [32, 33]. miR-634 has been reported to demonstrate anti-tumor activity by inhibition of cancer cell proliferation and migration [34-36]. Data from EdU staining and transwell assay showed that miR-634 suppressed Ang IIinduced HASMCs proliferation and migration, suggesting that miR-634 might be a potential target for the intervention of hypertension. Moreover, regulation of VSMCs differentiation [37] or contraction [38] participate in development of hypertension. The effect of miR-634 on VSMCs differentiation and contraction needs to be further investigated. Tissue macrophages play an important role in the pathogenesis of hypertension [39]. Macrophage-derived exosomes promoted the inflammation of endothelial cells, thus participating in hypertension [40]. Antibodies against endothelial cells mediated pulmonary arterial hypertension through regulation of endothelial cell apoptosis [41]. Since endothelial cells have been shown to release hyperpolarizing factors to modulate VSMCs and implicated in the pathophysiology of hypertension [42]. The regulatory role of miR-634 on other cells (endothelial cells, macrophages and immune cells) involved in hypertension should be investigated.

Prediction via Targetscan 7.2 (http://www.target scan.org/vert_72/) and validation by luciferase activity assay further confirmed that miR-634 could target Wnt4 in HASMCs. Moreover, miR-634 could decrease the expression of Wnt4. As reported before, Wnt4 was increased during proliferation process of VSMCs [43], and activation of Wnt pathway could contribute to VSMC proliferation and migration [44]. Therefore, inhibition of Wnt pathway could be useful for suppression of VSMCs proliferation and migration, thus attenuating hypertension [45]. Here, our results indicated that over-expression of Wnt4 promoted VSMCs proliferation and migration, and coun- teracted the suppressive effects of miR-634 on Ang IIinduced HASMCs proliferation and migration. These results suggested that miR-634 could attenuate hypertension through inhibition of Wnt pathway. Wnt could activate Disheveled and rescue $\beta$-catenin from destruction, and promote the nuclear translocation of $\beta$-catenin, thereby mediating renin-angiotensin system genes expression to regulate vascular remodeling or blood pressure during hypertension [46]. Data in this study showed that miR-634 increased cytoplasmic distribution of $\beta$-catenin, while overexpression of Wnt4 or in vitro Wnt4 treatment reversed the suppressive effect of miR-634 on nuclear distribution of $\beta$-catenin, suggesting that $\mathrm{Wnt} / \beta$-catenin pathway was involved in miR-634-mediated hypertension. In addition, non-canonical Wnt pathway was also found to be implicated in development of hypertension [47]. Whether noncanonical Wnt pathway was involved in miR-634-mediated hypertension remains to be investigated.

\section{Conclusions}

In general, our study for the first time demonstrated that miR-634 functioned as a novel regulator of VSMCs proliferation and migration via inhibiting Wnt $4 / \beta$ catenin pathway. As shown in Supplementary Fig. 4, miR-634 was down-regulated in Ang II-induced HASMCs, and over-expression of miR-634 suppressed proliferation and migration of Ang II-induced HASMCs through targeting of Wnt4. Over-expression of Wnt4 counteracted the suppressive effects of miR-634 on Ang II-induced proliferation and migration of HASMCs. This study provides new perspective for understanding the implication of miR634 during intervention of hypertension. However, the definite role of miR-634 in treatment of hypertension needs to be further confirmed in transgenic mice models with miR634 overexpression or silencing. Moreover, only one cell line (HASMCs) was used in this study, the effect of miR634 on other cells (endothelial cells, macrophages and immune cells), as well as the effect on interaction between HASMC and immune cells, should be investigated in further research.

\section{Author contributions}

LN and YK designed the study, supervised the data collection, analyzed the data, NS interpreted the data and prepare the manuscript for publication, LK and YX supervised the data collection, analyzed the data and reviewed the draft of the manuscript. All authors have read and approved the manuscript. 


\section{Ethics approval and consent to participate}

Ethical approval was obtained from the Ethics Committee of the Ethics Committee of the First Affiliated Hospital of Xi'an Jiaotong University (approval number: XJTU1AF2017LSL-001).

\section{Acknowledgment}

Not applicable.

\section{Funding}

This work was supported by the National Natural Science Foundation of China (Grant No. 81770426).

\section{Conflict of interest}

The authors declare no conflict of interest.

\section{Availability of data and materials}

All data generated or analyzed during this study are included in this published article.

\section{References}

[1] Sukhwinder K. Bhullar AKS, Naranjan S. Dhalla. Storeoperated calcium channels: Potential target for the therapy of hypertension. Reviews in Cardiovascular Medicine. 2019; 20: 139-151.

[2] Low PA, Tomalia VA. Orthostatic Hypotension: Mechanisms, Causes, Management. Journal of Clinical Neurology. 2015; 11: 220-226.

[3] Kokubo Y, Padmanabhan S, Iwashima Y, Yamagishi K, Goto A. Gene and environmental interactions according to the components of lifestyle modifications in hypertension guidelines. Environmental Health and Preventive Medicine. 2019; 24: 19.

[4] Arwood MJ, Cavallari LH, Duarte JD. Pharmacogenomics of Hypertension and Heart Disease. Current Hypertension Reports. 2015; 17: 586-586.

[5] Bobik A. The structural basis of hypertension: vascular remodelling, rarefaction and angiogenesis/arteriogenesis. Journal of Hypertension. 2005; 23: 1473-1475.

[6] Wang D, Uhrin P, Mocan A, Waltenberger B, Breuss JM, Tewari $\mathrm{D}$, et al. Vascular smooth muscle cell proliferation as a therapeutic target. Part 1: molecular targets and pathways. Biotechnology Advances. 2018; 36: 1586-1607.

[7] Wang D, Atanasov AG. The microRNAs Regulating Vascular Smooth Muscle Cell Proliferation: a Minireview. International Journal of Molecular Sciences. 2019; 20: 324.

[8] Fu X, Zhou Y, Cheng Z, Liao X, Zhou X. MicroRNAs: Novel Players in Aortic Aneurysm. BioMed Research International. 2016; 2015: 831641.

[9] Badawy H, Mesbah N, Abo-El-Matty D. A Brief review of Circulating MicroRNAs and Essential Hypertension. Records of Pharmaceutical and Biomedical Sciences. 2018; 2: 32-45.

[10] Liu Y, Taylor NE, Lu L, Usa K, Cowley AW, Ferreri NR, et al. Renal medullary microRNAs in Dahl salt-sensitive rats: miR$29 \mathrm{~b}$ regulates several collagens and related genes. Hypertension. 2010; 55: 974-982.
[11] Xin M, Small EM, Sutherland LB, Qi X, McAnally J, Plato CF, et al. MicroRNAs miR-143 and miR-145 modulate cytoskeletal dynamics and responsiveness of smooth muscle cells to injury. Genes \& Development. 2009; 23: 2166-2178.

[12] Sarrion I, Milian L, Juan G, Ramon M, Furest I, Carda C, et al. Role of circulating miRNAs as biomarkers in idiopathic pulmonary arterial hypertension: possible relevance of miR23a. Oxidative Medicine and Cellular Longevity. 2015; 2015: 792846.

[13] Li S, Zhu J, Zhang W, Chen Y, Zhang K, Popescu LM, et al. Signature microRNA expression profile of essential hypertension and its novel link to human cytomegalovirus infection. Circulation. 2011; 124: 175-184.

[14] Deb A. Cell-cell interaction in the heart via Wnt/ $\beta$-catenin pathway after cardiac injury. Cardiovascular Research. 2014; 102 : 214-223.

[15] Zheng Q, Chen P, Xu Z, Li F, Yi XP. Expression and redistribution of $\beta$-catenin in the cardiac myocytes of left ventricle of spontaneously hypertensive rat. Journal of Molecular Histology. 2013; 44: 565-573.

[16] Zhao Y, Wang C, Wang C, Hong X, Miao J, Liao Y, et al. An essential role for $\mathrm{Wnt} / \beta$-catenin signaling in mediating hypertensive heart disease. Scientific Reports. 2018; 8: 8996

[17] Cuevas CA, Tapia-Rojas C, Cespedes C, Inestrosa NC, Vio CP. B-Catenin-Dependent Signaling Pathway Contributes to Renal Fibrosis in Hypertensive Rats. BioMed Research International. 2015; 2015: 1-13.

[18] Xiao L, Xu B, Zhou L, Tan RJ, Zhou D, Fu H, et al. Wnt/ $\beta$ catenin regulates blood pressure and kidney injury in rats. Biochimica Et Biophysica Acta (BBA) - Molecular Basis of Disease. 2019; 1865: 1313-1322.

[19] Wei S, Wang Y, Zhang Q, Zhao S, Diao T, Li J, et al. Multiple Mechanisms are Involved in Salt-Sensitive HypertensionInduced Renal Injury and Interstitial Fibrosis. Scientific Reports. 2017; 7: 45952.

[20] Mosakhani N, Guled M, Lahti L, Borze I, Forsman M, Pääkkönen $\mathrm{V}$, et al. Unique microRNA profile in Dupuytren's contracture supports deregulation of $\beta$-catenin pathway. Modern Pathology. 2011; 23: 1544-1552.

[21] Agrawal R, Tran U, Wessely O. The miR-30 miRNA family regulates Xenopus pronephros development and targets the transcription factor Xlim1/Lhx1. Development. 2009; 136: $3927-$ 3936.

[22] Zou X, Wang J, Chen C, Tan X, Huang Y, Jose PA, et al. Secreted Monocyte miR-27a, via Mesenteric Arterial Mas ReceptoreNOS Pathway, Causes Hypertension. American Journal of Hypertension. 2020; 33: 31-42.

[23] Feihl F, Liaudet L, Levy BI, Waeber B. Hypertension and microvascular remodelling. Cardiovascular Research. 2008; 78: 274-285.

[24] Wang P, Xu J, Hou Z, Wang F, Song Y, Wang J, et al. MiRNA34a promotes proliferation of human pulmonary artery smooth muscle cells by targeting PDGFRA. Cell Proliferation. 2016; 49: 484-493.

[25] Jin Y, Jin Y, Chen B, Tipple TE, Nelin LD. Arginase II is a target of miR-17-5p and regulates miR-17-5p expression in human pulmonary artery smooth muscle cells. American Journal of Physiology. Lung Cellular and Molecular Physiology. 2014; 307: L197-L204.

[26] Zeng Y, Zhang X, Kang K, Chen J, Wu Z, Huang J, et al. MicroRNA-223 Attenuates Hypoxia-induced Vascular Remodeling by Targeting RhoB/MLC2 in Pulmonary Arterial Smooth Muscle Cells. Scientific Reports. 2016; 6: 24900.

[27] Marques FZ, Booth SA, Charchar FJ. The emerging role of noncoding RNA in essential hypertension and blood pressure regulation. Journal of Human Hypertension. 2015; 29: 459-467.

[28] Li F, Zhang C, Luo X, Peng J, Yang T. Involvement of the MiR181b-5p/HMGB1 Pathway in Ang II-induced Phenotypic Trans- 
formation of Smooth Muscle Cells in Hypertension. Aging and Disease. 2019; 10: 231-248.

[29] Ren X, Tong Y, Ling L, Chen D, Sun H, Zhou H, et al. NLRP3 Gene Deletion Attenuates Angiotensin II-Induced Phenotypic Transformation of Vascular Smooth Muscle Cells and Vascular Remodeling. Cellular Physiology and Biochemistry. 2017; 44: 2269-2280.

[30] Te Riet L, van Esch JHM, Roks AJM, van den Meiracker AH, Danser AHJ. Hypertension: renin-angiotensin-aldosterone system alterations. Circulation Research. 2015; 116: 960-975.

[31] Wu W, Hu C, Chen X, Zhang W, Li X, Xiong X, et al. MicroRNA-130a mediates proliferation of vascular smooth muscle cells in hypertension. American Journal of Hypertension. 2011; 24: 1087-1093.

[32] Xu T, Zhu H, Li D, Lang Y, Cao L, Liu Y, et al. Luteolin Inhibits Angiotensin II-Stimulated VSMC Proliferation and Migration through Downregulation of Akt Phosphorylation. EvidenceBased Complementary and Alternative Medicine. 2015; 2015: 931782.

[33] Yu S, Chen Y, Chen S, Ye N, Li Y, Sun Y. Klotho Inhibits Proliferation and Migration of Angiotensin II-Induced Vascular Smooth Muscle Cells (VSMCs) by Modulating NF-kB p65, Akt, and Extracellular Signal Regulated Kinase (ERK) Signaling Activities. Medical Science Monitor. 2018; 24: 4851-4860.

[34] Guo J, Zhang C, An J, Xiao Y, Shao S, Zhou N, et al. Expression of miR-634 in gastric carcinoma and its effects on proliferation, migration, and invasion of gastric cancer cells. Cancer Medicine. 2018; 7: 776-787.

[35] Zhang CZ, Cao Y, Fu J, Yun J, Zhang M. MiR-634 exhibits antitumor activities toward hepatocellular carcinoma via Rab1a and DHX33. Molecular Oncology. 2016; 10: 1532-1541.

[36] Cong J, Liu R, Wang X, Jiang H, Zhang Y. MiR-634 decreases cell proliferation and induces apoptosis by targeting mTOR signaling pathway in cervical cancer cells. Artificial Cells, Nanomedicine, and Biotechnology. 2016; 44: 16941701.

[37] Owens GK, Kumar MS, Wamhoff BR. Molecular regulation of vascular smooth muscle cell differentiation in development and disease. Physiological Reviews. 2004; 84: 767-801.

[38] Touyz RM, Alves-Lopes R, Rios FJ, Camargo LL, Anagnostopoulou A, Arner A, et al. Vascular smooth muscle contraction in hypertension. Cardiovascular Research. 2018; 114: 529-539.

[39] Justin Rucker A, Crowley SD. The role of macrophages in hypertension and its complications. Pflugers Archiv: European Journal of Physiology. 2017; 469: 419-430.

[40] Osada-Oka M, Shiota M, Izumi Y, Nishiyama M, Tanaka M, Yamaguchi T, et al. Macrophage-derived exosomes induce inflammatory factors in endothelial cells under hypertensive conditions. Hypertension Research. 2017; 40: 353-360.
[41] Li C, Liu P, Song R, Zhang Y, Lei S, Wu S. Immune cells and autoantibodies in pulmonary arterial hypertension. Acta Biochimica et Biophysica Sinica. 2017; 49: 1047-1057.

[42] Puddu P, Puddu GM, Zaca F, Muscari A. Endothelial dysfunction in hypertension. Acta Cardiologica. 2000; 55: 221-232.

[43] Tsaousi A, Williams H, Lyon CA, Taylor V, Swain A, Johnson $\mathrm{JL}$, et al. Wnt $4 / \beta$-Catenin Signaling Induces VSMC Proliferation and is Associated with Intimal Thickening. Circulation Research. 2011; 108: 427-436.

[44] Marchand A, Atassi F, Gaaya A, Leprince P, Le Feuvre C, Soubrier F, et al. The Wnt/beta-catenin pathway is activated during advanced arterial aging in humans. Aging Cell. 2011; 10: 220-232.

[45] Alapati D, Rong M, Chen S, Lin C, Li Y, Wu S. Inhibition of LRP5/6-mediated Wnt/beta-catenin signaling by Mesd attenuates hyperoxia-induced pulmonary hypertension in neonatal rats. Pediatric Research. 2013; 73: 719-725.

[46] Vallee A, Levy BL, Blacher J. Interplay between the reninangiotensin system, the canonical WNT/beta-catenin pathway and PPARgamma in hypertension. Current Hypertension Reports. 2018; 20: 62.

[47] Abou Ziki MD, Mani A. Wnt signaling, a novel pathway regulating blood pressure? State of the art review. Atherosclerosis. 2017; 262: 171-178.

Supplementary material: Supplementary material associated with this article can be found, in the online version, at https:/www.fbscience.com/Landmark/articles/10. 52586/4953.

Abbreviations: miRNAs, microRNAs; Ang, angiotensin; UTR, untranslated region.

Keywords: HASMCs; miR-634; Migration; Proliferation; Wnt4/ $\beta$-catenin; Hypertension

Send correspondence to: Yuming Kang, Department of Physiology and Pathophysiology, Xi'an Jiaotong University School of Basic Medical Sciences, Shaanxi Engineering and Research Center of Vaccine, Key Laboratory of Environment and Genes Related to Diseases of Education Ministry of China, 710061 Xi'an, Shaanxi, China, E-mail: ykang@mail.xjtu.edu.cn 